\title{
Screening and diagnosis of hyperthyroidism: an attempt at test reduction
}

\author{
P. FRAGU', A. AlPÉROVITCH ${ }^{2}$, AND E. PATOIS ${ }^{2}$ \\ From 'l'Unité de Médecine Nucléaire and 'l'Unité de Recherches sur les Méthodes Statistiques et \\ Épidémiologiques et leurs applications à l'étude des Maladies Chroniques, Villejuif
}

SUMmARY A sequential strategy for the diagnosis of hyperthyroidism has been prospectively appraised on 410 patients using a pocket calculator-aided diagnostic system. It was found that for $64 \%$ of the patients final diagnosis could be established from nine clinical signs, ankle jerk time and free thyroxin index. For the $36 \%$ of doubtful subjects, $T_{3}$ determination permitted the reduction of uncertainty to $9 \%$. No misdiagnosis was observed. By comparing this strategy with the physician's usual diagnosis process, in which all clinical signs and several thyroid function tests were used, it appeared that the number of tests was reduced by $65 \%$ for $T_{3}$ requests and by $70 \%$ for other tests ( ${ }^{99 m}$ Tc uptake and TRH). The cost-saving was estimated to be about $28 \%$. The interest of this calculator-aided decision model resides in the possibility for the general practitioner to refer only doubtful and hyperthyroid subjects to a thyroid unit.

The ruling strategy for diagnosis of hyperthyroidism is at present based on a complete clinical examination accompanied by two in vitro thyroid function tests: free thyroxin $\left(T_{4}\right)$ index and $T_{3}$ level (Harvey, 1971; Tubiana et al., 1972; Vagenakis et al., 1976). In most cases this efficient battery of investigations allows the specialist to confirm the diagnosis with certainty.

The specialist has confidence in his diagnosis only when there is total agreement between his clinical assessment and his judgment on the results of the routine laboratory tests. If they do not entirely agree he will request follow-up or therapeutic tests before reaching a conclusion. If it were possible to succeed in establishing some objective rules based on the specialist's diagnostic reasoning, positive consequence could then be derived.

Firstly, if objective diagnostic rules could be transmitted, particularly to a GP, they could help him to handle alone many of the patients he formerly referred to a specialist. This extension of the GP's role would involve important time-saving for both the patient and the specialist without adding substantially to the GP's work.

Secondly, a definition of these objective rules might be helpful in reducing the number of investigations requested. Indeed, in most 'thyroid units', a large percentage of requests are for screening (Brittonet al., 1975). Although the cost per patient of a systematic request for both free $T_{4}$ index and $T_{3}$ measurement is not very great, the overall expense is large. With this in mind, it was of interest to investigate the necessity of systematically requesting both of these in vitro thyroid function tests, $T_{3}$ measurement being particularly questionable at the screening step of the diagnostic strategy.

The aim of this study was to define and assess a sequential strategy for the detection of hyperthyroidism supported by a simple pocket calculator-aided decision programme.

\section{Material and methods}

From all subjects referred by GPs for a possible hyperthyroid problem, two categories of patients were excluded: firstly, those previously treated for a thyroid disease, and, secondly, those with abnormal thyroid palpation when toxic adenoma was suspected after a thyroid scan.

\section{Design of the calculator-aided diagnosis strategy}

A simple Bayesian not 'computer-aided' but 'pocket calculator-aided' diagnosis programme was used to simulate the reasoning analysis of the specialist.

1. As an objective substitute for subjective clinical impression 'clinical probability' of hyperthyroidism was calculated using the following nine signs: upper lid retraction, pulse $90 / \mathrm{min}$, weight loss $>5 \mathrm{~kg}$, changes in appetite and/or thirst, heat tolerance, warm moist skin, nervousness, and fine finger tremor. 
2. Likewise, the biological assessment based upon the 'biological probability' of hyperthyroidism was calculated from laboratory test results. The frequencies of the different clinical profiles and the distributions of laboratory test results for euthyroid and hyperthyroid patients have been estimated in a previous study (Alpérovitch et al., 1977).

The 'computer' was programmed* to calculate these probabilities from data introduced by the clinician for clinical profile, free thyroxin $\left(T_{4}\right)$ index, and $\mathrm{T}_{3}$.

Two cut-off points were determined for each probability in order to define three categories: euthyroid-uncertainty-hyperthyroid. These cut-off points were respectively 0.20 and 0.80 for the clinical probability of hyperthyroidism and 0.10 and 0.90 for the biological probability of hyperthyroidism.

The proposed decision rule was to terminate only if the two probabilities were either both in the euthyroid zone or both in the hyperthyroid zone. If not, it was advisable to request further investigations.

\section{Assessment of the proposed diagnostic strategy}

The proposed diagnostic strategy was assessed on a sample of 410 patients (298 euthyroid and 112 hyperthyroid). Four clinicians were involved in this study. The thyroid status of each patient was determined by a specialist from a complete clinical examination, including measurement of ankle jerk time when available, and all tests that he deemed it advisable to request: $T_{4}$ and $T_{3}$ serum levels, $T_{3}$ resin uptake ratio and free thyroxin index were requested for all patients. For 103 patients, the radioactive uptake by the thyroid gland at 20 minutes for ${ }^{99 \mathrm{~m}} \mathrm{Tc}$ was also measured (Aubert et al., 1977). In addition. for 40 patients, Werner's test, or TRH test, or a short follow-up, or a therapeutic test were required to obtain a reliable diagnosis. The diagnosis decided on by the specialist at the end of this investigation was considered as the true diagnosis.

With the calculator-aided programme, it was easy to simulate for each patient the diagnostic path corresponding with the decision rules given in the previous section. Thus, it was possible to compare for

-A detailed description of the pocket calculator programme can be sent on request. the same patients: (1) the number of requests for the various tests with that observed by the usual diagnostic procedures, and (2) the conclusion proposed at the end of the simulated diagnostic path with that stated by the clinician.

\section{Results}

In order to define the best sequential strategy, the following three questions were more closely investigated.

1. Is it possible to manage safe screening from basic clinical profile and free thyroxin index? And what is the efficacy of this screening?

2. What is the value of the systematic measurement of ankle jerk time at this screening step?

3. What test strategy should be proposed after the screening-oriented step?

Screening from clinical examination and free thyroxin index

Applying the decision rules defined from these two probabilities of hyperthyroidism it was possible to manage a preliminary screening.

For $61 \%$ of the patients ( 252 out of 410 ), a clinical examination and free thyroxin $\left(T_{4}\right)$ index wereo sufficient to answer the diagnostic problem. In all these cases, the proposed conclusion was identical with the diagnosis given by the clinician from the results of the battery of tests that he had selected. The screening efficacy was particularly high for euthyroid subjects, for $73 \%$ of whom the diagnostic problem was solved at this screening step. For $70 \%$ of the hyperthyroid patients, further tests were necessary for a confident decision.

\section{Usefulness of ankle jerk time}

When technically possible, ankle jerk time was measured, and this measurement was considered as an extension of the usual clinical examination.

For 340 of the 410 patients, these data were available (Table 2) and the addition of ankle jerk time measurement slightly improved the clinical evaluation. A definitive conclusion was obtained for $67 \%$ of the subjects, instead of $63 \%$ without ankle jerk time. This difference is significant (Table 2) and the benefit is particularly clear for hyperthyroid patients (Table 1).

Table 1 Evaluation of the diagnosis strategy using the calculator-aided decision programme

\begin{tabular}{|c|c|c|c|c|c|c|c|c|}
\hline \multirow{4}{*}{$\begin{array}{l}\text { Conclusion of calculator-aided decision programme } \\
\text { Clinical profile }+ \text { free } T_{4} \text { index } \\
\text { Clinical profile }+ \text { free } T_{4} \text { index }+ \text { ankle jerk } \\
\text { Clinical profile }+ \text { free } T_{4} \text { index }+ \text { ankle jerk }+T_{2}\end{array}$} & \multicolumn{6}{|c|}{ Specialist diagnosis } & & \\
\hline & \multicolumn{3}{|c|}{ Euthyroid (298 cases) } & \multicolumn{3}{|c|}{ Hyperthyroid (112 cases) } & \multirow{2}{*}{\multicolumn{2}{|c|}{$\begin{array}{l}\text { Correct } \\
\text { confident } \\
\text { Diagnosis }\end{array}$}} \\
\hline & $E \boldsymbol{u}$ & Uncertain & Hyper & $E u$ & Uncertain & Hyper & & \\
\hline & $\begin{array}{l}217 \\
213 \\
293 \\
247\end{array}$ & $\begin{array}{r}81 \\
85 \\
5 \\
51\end{array}$ & $\begin{array}{l}0 \\
0 \\
0 \\
0\end{array}$ & $\begin{array}{l}0 \\
0 \\
0 \\
6\end{array}$ & $\begin{array}{l}77 \\
61 \\
33 \\
80\end{array}$ & $\begin{array}{l}35 \\
51 \\
79 \\
26\end{array}$ & $\begin{array}{l}252 \\
264 \\
372 \\
273\end{array}$ & $\begin{array}{l}(61.5 \%) \\
(64.4 \%) \\
(90.7 \%) \\
(66.6 \%)\end{array}$ \\
\hline
\end{tabular}


Table 2 Improvement of the screening efficiency of the programme when ankle jerk time was included in clinical evaluation

\begin{tabular}{|c|c|c|c|}
\hline $\begin{array}{l}\text { Scre } \\
\text { Screening } \\
\text { conclusion without } \\
\text { ankle jerk time }\end{array}$ & Confident & Uncertain & Total \\
\hline $\begin{array}{l}\text { Confident } \\
\text { Uncertain } \\
\text { Total }\end{array}$ & $\begin{array}{l}206 \\
23 \\
229(67 \%)\end{array}$ & $\begin{array}{r}7 \\
104 \\
111\end{array}$ & $\begin{array}{l}213(63 \%) \\
127 \\
340\end{array}$ \\
\hline
\end{tabular}

$\chi^{2}$ (paired cases) $=8.53 \quad P=<0.01$

What test strategy to use after the screening-oriented step?

The problem of hyperthyroid patients with normal $\mathrm{T}_{3}$ measurement has been pointed out by many authors. So, in our study, $\mathrm{T}_{3}$ assay was not a reliable preliminary screening method when used with clinical signs because it gave rise to misdiagnosis of at least $5 \%$ of hyperthyroid cases. But when used as a secondary test, it may help to increase discrimination. For a given patient, the starting point of this second step was a 'post-screening probability' of hyperthyroidism calculated both from clinical examination including ankle jerk time if available, and from free thyroxin index.

This probability was compared with the probability calculated from $\mathrm{T}_{3}$ assay. For $74 \%$ of the doubtful patients (108 out of 146), a confident conclusion was possible after the $T_{3}$ assay (Table 3 ). For the other cases, which represent $9 \%$ of the whole sample, the usefulness of the TRH test and of the thyroid uptake

Table 3 Value of $\mathrm{T}_{3}$ concentration measurement in doubtful cases

\begin{tabular}{lllll}
\hline Clinician's final diagnosis & \multicolumn{2}{l}{ Euthyroid: 85 cases } & Hyperthyroid: 61 cases \\
\hline Conclusion of calculator- & & & & \\
aided diagnostic strategy & Euthyroid & 80 & Euthyroid & 0 \\
& Uncertain & 5 & Uncertain & 33 \\
& Hyperthyroid & 0 & Hyperthyroid & 28 \\
\hline
\end{tabular}

These results concern 146 of the 410 patients; 264 patients (64\%) were classified at the screening-oriented step. of ${ }^{99 \mathrm{~m}} \mathrm{Tc}$ should be carefully investigated, particularly because we observed a high percentage $(10 \%)$ of hyperthyroid patients with uptakes of ${ }^{99 \mathrm{~m}} \mathrm{Tc}$ in the euthyroid range.

\section{Comments and discussion}

\section{Overall evaluation}

The results of this study indicate that the calculator-aided diagnostic strategy seems highly advisable for patients presenting with a possible hyperthyroidism problem. The screening-oriented step based on clinical evaluation including measurement of ankle jerk time and free thyroxin index permits a definite answer to the diagnosis problem for $64 \%$ of the patients. For doubtful cases, $\mathrm{T}_{3}$ concentration is then measured on a preserved blood sample. After this second test, diagnosis is made for $91 \%$ of the patients, and only $9 \%$ of the patients need further investigation. This approach improves the clinician's efficacy because it confers important savings in cost, particularly for euthyroid subjects. By comparing this strategy with the usual process of diagnosis, it appears that the number of $T_{s}$ requests is reduced by $65 \%$ and the number of other tests $\left({ }^{99 m}\right.$ Tc uptake and TRH) by $70 \%$. The average cost of the diagnostic process would be reduced by $30 \%$ for euthyroid subjects and by $23 \%$ for hyperthyroid subjects (Table 4). The expected benefit of this sequential strategy is closely related to the percentage of hyperthyroid cases in the screened population: the smaller this percentage, the greater the benefit of the proposed diagnostic strategy. In a population less than $40 \%$ hyperthyroid, this approach appears valid.

\section{GPs' screening role}

Since the diagnostic programme is written for a simple calculator, it is not unrealistic to anticipate that this programme could play the role of an efficient GP's assistant for the management of a high percentage of patients. As shown in Table 1, the percentage of patients with both 'clinical probability' and 'free thyroxin index probability' in the euthyroid

\begin{tabular}{|c|c|c|c|c|}
\hline & \multicolumn{4}{|c|}{ Number of test requests by } \\
\hline & \multicolumn{2}{|c|}{ Calculator-aided diagnostic strategy } & \multicolumn{2}{|c|}{ Specialist } \\
\hline & Euthyroid & Hyperthyroid & Euthyroid & Hyperthyroid \\
\hline Free thyroxin index & 298 & 112 & 298 & 112 \\
\hline Ta measurement & 85 & 61 & 298 & 112 \\
\hline Other tests $\left(99^{\mathrm{m}} \mathrm{Tc}\right.$ uptake and TRH test) & 5 & 33 & 59 & 64 \\
\hline Average cost per subject in French franes & 256 & 337 & 364 & 438 \\
\hline Saving per patient in French francs & 108 & 101 & - & - \\
\hline $\begin{array}{l}\text { Cost-saving per year in French francs for } 600 \text { new patients } \\
\text { of whom } 75 \% \text { are euthyroid }\end{array}$ & \multicolumn{4}{|c|}{63750} \\
\hline
\end{tabular}


zone is about $50 \%$. Half of these patients have abnormal thyroid palpation and must endure further investigations. But it was not necessary for the others to be referred to the specialist, and the number of consultations in the specialised unit might consequently be reduced by at least $25 \%$.

A problem must be solved beforehand. None of the nine clinical signs used by the programme was noted to be present in $15 \%$ of the patients referred by the GP for a possible hyperthyroidism at the time when they were examined by the specialist. This discrepancy might be due to various facts, particularly variability of some signs and observer error. In order to identify and to reduce this discrepancy, a co-operative study with a GP has been undertaken.

\section{Strategy transferability}

The proposed diagnostic strategy simulates the clinician's reasoning, with the cut-off points representing subjective decision rules of a skilled clinician. The fact that they are based on probabilities (euthyroid or hyperthyroid) makes them more general than rules based on ranges defined by laboratory measurements (Britton, 1975).

The programme parameters (a priori probability of hyperthyroidism, frequencies of each clinical sign for euthyroid and hyperthyroid subjects, means and standard deviations for laboratory tests) must be updated because of the variations from one hospital to another and also within the same hospital, as they can change with time. This updating does not present real difficulties, because of the simplicity of our system.

In conclusion, we should emphasise that this experiment is one of the few studies in which so called 'computer'-aided medical decision offers an approach capable of playing a major role in daily medical practice rather than a simple academic exercise (De Dombal et al., 1972; Anderson et al., 1976; Alpérovitch and Fragu, 1977). In addition, it can result in savings in time and cost, because it requires no more than a pocket calculator and the medical competence of a general practitioner.

We thank Professors M. Tubiana and J. Lellouch, Dr. S. Askienazy, P. Charbord, P. Gardet, C. Parmentier, and J. Zalokar.

Reprints from Dr. A. Alpérovitch, Institut National de la Santé et de la Recherche Médicale, 16 bis, avenue Paul Vaillant-Couturier, 94800 Villejuif, France.

\section{References}

Alpérovitch, A., and Fragu, P. (1977). A suggestion for an effective use of a computer-aided diagnosis system in screening for hyperthyroidism. Methods of Information in Medicine, 16, 93-95.

Alpérovitch, A., Fragu, P., Patois, E., and Lellouch, J. (1977). Routine use of computer-aided decision system. A positive evaluation in hyperthyroidism screening. In Medinfo 77, p. 209. Edited by D. B. Shires and H. Wolf. North-Holland Publishing Company: Amsterdam.

Anderson, G., Llorena, C., Davidson, D., and Taylor, T. R. (1976). Practical application of computer-assisted $\mathrm{N}$ decision making in an antenal clinic. A feasibility study $\vec{O} \overrightarrow{\mathrm{G}}$ Methods of Information in Medicine, 15, 224-229.

Aubert, B., Chavaudra, J., Di Paola, R., Fragu, P., and Bok B. (1977). Détermination automatique de la fixation de $\$$ radionucléides dans le organes à partir de scintigraphies $\mathbb{D}$ numériques. Application à la thyroïde. Journal Français de la Biophysique et Médecine Nucléaire, 1, 101-108.

Britton, K. E., Quinn, V., Brown, B. L., and Ekins, R. P (1975). A strategy for thyroid function tests. British Medical Journal, 3, 350-352.

De Dombal, F. T., Leaper, D. J., Staniland, J. R., McCann A. P., Horrocks, J. C. (1972). Computer-aided diagnosis of acute abdominal pain. British Medical Journal, 2, 9-13.

Harvey, E. D. (1971). Indice of thyroid function in thyrotoxicosis. Lancet, 2, 230-233.

Tubiana, M., Fragu, P., and Bazin, J. P. (1972). Indications relatives des tests in vivo et in vitro pour l'exploration de la fonction thyroïdienne. In 14ème Colloque de Médecine Nucléaire de langue française. Edited by T. Planiol. Revue de Médecine de Tours: France.

Vagenakis, A. G., Braverman, L. E. (1976). Thyroid function test-which one? Annals of Internal Medicine, 84, 607-608. 